\title{
LIPSCHITZ APPROXIMATION OF HOMEOMORPHISMS
}

\author{
PEKKA TUKIA
}

\section{Results}

1. The aim of this paper is to prove a modification of Theorem 2.4 of Väisälä [5], and to prove, using this modification, the LIP hauptvermutung for $n \leqq 3$.

This paper is directly based on Väisälä [5]. We assume the knowledge of [5] (only Sections 1 and 2 are needed) and use freely its definitions and notations.

Let $f: X \rightarrow Y$ be a map between metric spaces and let $x \in X$. We say that $f$ is locally bilipschitz at $x$ if there is a neighbourhood $U$ of $x$ such that $f \mid U$ is a homeomorphism $U \rightarrow f(U)$ with bilip $(f \mid U)<\infty$. If $A \subset X$, we say that $f$ is locally bilipschitz in $A$ if $f$ is locally bilipschitz at $x$ for every $x \in A$.

2. We begin by proving (cf. Theorem 2.4 of [5] and Theorem 3 below).

Theorem 1. Let $n \leqq 3$ and $\varepsilon>0$. Let $f: I^{n} \rightarrow R^{n}$ be an embedding. Then there is an embedding $f^{*}: I^{n} \rightarrow R^{n}$ such that

(1) $f^{*}\left|\partial I^{n}=f\right| \partial I^{n}$

(2) $f^{*} \mid$ int $I^{n}$ is PL,

(3) $\left\|f^{*}-f\right\|<\varepsilon$.

In addition, assume that $f$ is locally bilipschitz in a closed set $C \subset \partial I^{n}$. Then we may assume that $f^{*}$ is locally bilipschitz in $C$.

Thus $f^{*}$, being $\mathrm{PL}$ in int $I^{n}$, is locally bilipschitz in $C \cup$ int $I^{n}$. This observation is crucial for the following LIP hauptvermutung for $n \leqq 3$ :

Theorem 2. Let $n \leqq 3$ and let $f: M \rightarrow N$ be a homeomorphism between two LIP $n$-manifolds. Let $\varepsilon: M \rightarrow(0, \infty)$ be continuous. Then there is a lipeomorphism $g: M \rightarrow N$ such that $d(f(x), g(x))<\varepsilon(x)$ for $x \in M$. Here $d$ is the metric of $N$.

In addition, if $f \mid U$ is a lipeomorphism for some open set $U \subset M$ and if $C \subset U$ is closed, we may assume $f|C=g| C$. 
Since every paracompact $n$-manifold, $n \leqq 3$, has a PL-structure which induces on it a LIP-structure, Theorem 2 has the following

Corollary. Every LIP n-manifold, $n \leqq 3$, is lipeomorphic to a PL manifold.

This was proved in [5] for $n \leqq 2$ by a different method (Theorem 3.8) and the LIP hauptvermutung was obtained as a corollary for $n=2$.

Finally we show, using Theorem 2, that a slightly more general version of Theorem 1 is true:

Theorem 3. Let $n, \varepsilon$, and $f$ be as in Theorem 1. Then we may assume that $f^{*}$ of Theorem 1 is locally bilipschitz at all points of $\partial I^{n}$ where $f$ is locally bilipschitz.

Since Theorem 1 seems quite satisfactory for most purposes, our proof of Theorem 3 is rather sketchy.

3. The idea underlying Theorem 2 was used in Sorvali [3, Theorem 2.1] to show that if two compact Riemann surfaces are homeomorphic, then there is a quasiconformal homeomorphism between them. The proof was based on Riemann's mapping theorem and the Beurling-Ahlfors extension of a homeomorphism $f: R \rightarrow R$ to a homeomorphism $F$ of $U=\{z \in C: \operatorname{Im} z>0\}$. If $f$ is quasisymmetric, $F$ is quasiconformal, but in any case $F$ is locally quasiconformal. Originally, the idea was due to O. Lehto.

4. Quasiconformal versions of the above theorems can most probably be proved by the same method.

5. I wish to thank J. Luukkainen and J. Väisälä for pointing out errors in the earlier versions of this paper.

6. Sullivan [4] has proved the LIP hauptvermutung for $n \neq 4$. His methods are much more advanced than ours, which are quite elementary, although our proofs are unduly complicated. In addition to the results of [5] already mentioned, the LIP hauptvermutung was proved in Luukkainen-Väisälä [1, Section 8] for a space homeomorphic either to $R^{n}, S^{n}$, or $I^{n}$ with some restrictions on $n$.

\section{Proofs}

We assume $n=2$ or $n=3$ since proofs are trivial if $n=1$.

7. We begin by establishing a lemma needed in the proofs of Theorems 1 and 3 .

Let $R_{0}=I^{n}$ and $R_{k}=I^{n} \backslash$ int $I^{n}\left(1-2^{2-k} / 3\right), k \geqq 1$. Then, if $k \geqq 1, R_{k}$ is a ringlike subset of $I^{n}$ and we obtain $R_{k+1}$ from $R_{k}$ by dividing $R_{k}$ into two rings and discarding the inner one. There is a natural way of dividing $R_{k}$ into cubes of side length $2^{2-k} / 3$ if $k \geqq 1$ and of length 2 if $k=0$ in such a way that, for each cube $Q$, $Q \cap \operatorname{int} I^{n}$ is a union of cubes of $K$ (cf. the figure in [5]). We denote the family of these cubes into which we have divided each $R_{k}$ by $\bar{K}_{k}, k \geqq 0$. 
Let $M$ be an $n$-submanifold of $I^{n}$. We say that $M$ is of finite $K$-type if $M \cap \operatorname{int} I^{n}$ is a union of cubes of $K$ and if there is $k \geqq 0$ such that $M \cap R_{k}$ is a union of cubes of $\bar{K}_{k}$. Therefore $M$ is a finite union of cubes of $K \cup \bar{K}_{k}$ for sufficiently big $k$. Let $\mathscr{F}$ be a family of subsets of $I^{n}$ and $X \subset I^{n}$. We often need to consider a family of sets of the form $\{S \in \mathscr{F}: S \subset X\}$ which we therefore denote by $\mathscr{F} \mid X$.

Lemma. Let $C \subset \partial I^{n}$ be compact and contained in some face $F$ of $I^{n}$ or let $C=\partial I^{n}$. Then there is a sequence $M_{0}=I^{n} \supset M_{1} \supset M_{2} \supset \ldots$ of submanifolds of $I^{n}$ that are of finite $K$-type such that:

(i) $\bigcap_{i \geqq 0} M_{i}=C$.

(ii) $M_{i+1} \subset$ int $M_{i}$ (int is relative to $I^{n}$ ), $i \geqq 0$.

(iii) $\operatorname{cl}\left(\partial M_{i} \cap \operatorname{int} I^{n}\right) \cap C=\emptyset$ for $i \geqq 0$.

(iv) Each $M_{i}$ is a union of cubes $Q \in K_{j}(F)=\left\{Q \in \bar{K}_{j}: Q \cap F \neq \emptyset\right\}$ for some $j \geqq 0$, or, if $C=\partial I^{n}, M_{i}=R_{i}$.

(v) Let $X$ be either $M_{i}$ or $\operatorname{cl}\left(M_{i} \backslash M_{i+1}\right)$ for some $i \geqq 0$. Then there is a partition $K_{1}(X) \cup \ldots \cup K_{m}(X)$ of $K \mid X$ ( $m$ depends on $X$ ) such that if $K_{r}^{*}(X)=K_{1}(X) \cup \ldots$ $\cup K_{r}(X), F_{r}(X)=\cup K_{r}^{*}(X)$ and $P_{Q}(X)=2 I^{n} \cap \alpha_{Q}^{-1} F_{r-1}(X)$ for $Q \in K_{r}(X)$ (cf. [5, 2.7]), then the conditions (1)-(3) of $[5,2.7]$ remain true after the substitutions $K_{r} \mapsto K_{r}(X)$ and $P_{Q^{\mapsto}} \mapsto P_{Q}(X)$.

(vi) Let $X$ be $\operatorname{cl}\left(M_{i} \backslash M_{i+1}\right)$ for some $i \geqq 0$. Let $K_{0}^{\prime}(X)=K \mid\left(\operatorname{cl}\left(M_{i-1} \backslash M_{i}\right) \cup\right.$ $\left.\operatorname{cl}\left(M_{i+1} \backslash M_{i+2}\right)\right) \quad$ if $\left.i=0, M_{-1}=\emptyset\right)$. Then there is a partition $K_{1}^{\prime}(X) \cup \ldots \cup K_{m^{\prime}}^{\prime}(X)$ of $K \mid X\left(m^{\prime}\right.$ depends on $\left.X\right)$ such that if $K_{r}^{\prime *}(X)=K_{0}^{\prime}(X) \cup \ldots \cup K_{r}^{\prime}(X), F_{r}^{\prime}(X)=\cup K_{r}^{\prime *}(X)$ and $P_{Q}^{\prime}(X)=2 I^{n} \cap \alpha_{Q}^{-1} F_{r-1}^{\prime}(X)$ for $Q \in K_{r}^{\prime}(X)$, then the conditions (1)-(3) for $r \geqq 1$ of $[5,2.7]$ remain true after the substitution $K_{r} \mapsto K_{r}^{\prime}(X)$ and $P_{Q} \mapsto P_{Q}^{\prime}(X)$.

Proof. It is easy to see that we can find a sequence $M_{0} \supset M_{1} \supset \ldots$ satisfying conditions (i) - (iv). In (v) and (vi) we need not care about the condition (3) of $[5,2.7]$ since if we can find $K_{i}(X)$ and $K_{i}^{\prime}(X)$ that satisfy (1) and (2) we can always have (3) by further partitioning of the families $K_{i}(X)$ and $K_{i}^{\prime}(X), i \geqq 1$. Also, we assume $n=3$ since the proof is much simpler if $n=2$.

First we note the following fact. Let $Y$ be either a cube $Q \in \bar{K}_{l}, l \geqq 0$, or of the form $\operatorname{cl}\left(Q \backslash\left(\cup K_{k}(F)\right)\right)$ or $\operatorname{cl}\left(Q \backslash R_{k}\right)$ where $Q \in \bar{K}_{l}$ and $k>l$. Then $K_{1}\left|Y, \ldots, K_{M}\right| Y$ is a partition of $K \mid Y$ satisfying conditions (1) and (2) of [5, 2.7], modified as in (v). This fact is fundamental to our proof of (v) and (vi).

The manifold $X$ is a finite union $\left(\cup K_{X}^{\prime}\right) \cup\left(\cup K_{X}^{\prime \prime}\right)$ where $K_{X}^{\prime}=K_{k}(F) \mid X$ (if $\left.C=\partial I^{n}, K_{X}^{\prime}=\bar{K}_{k} \mid X\right)$ and $K_{X}^{\prime \prime}=\left\{\operatorname{cl}\left(Q \backslash\left(\cup K_{k}(F)\right)\right): Q \in K_{l}(F)\right\} \mid X$ (if $C=\partial I^{n}, \quad K_{X}^{\prime \prime}=$ $\left.\left\{\mathrm{cl}\left(Q \backslash R_{k}\right): Q \in \bar{K}_{l}\right\}\right)$ for some $k>l \geqq 0$ (or $k=0$ and $K_{X}^{\prime \prime}=\emptyset$ ). We show that we can arrange the elements of $K_{X}^{\prime} \cup K_{X}^{\prime \prime}$ in a sequence, $K_{X}^{\prime} \cup K_{X}^{\prime \prime}=\left\{Q_{0}, \ldots, Q_{s-1}\right\}$, in such a way that if

$$
\text { (*) } K_{i M+j}(X)=K_{j} \mid Q_{i} \quad\left(K=K_{1} \cup \ldots \cup K_{M}\right) \text {, }
$$


then $K_{1}(X), \ldots, K_{s M}(X)$ is a partition of $K \mid X$ satisfying (v). In fact, it suffices for this that the sequence $\left\{Q_{0}, \ldots, Q_{s-1}\right\}$ satisfies the following condition, analogous to $(2)$ of $[5,2.7]$.

(**) For $r<s, \bigcup_{i=0}^{r} Q_{i}$ is an n-manifold and $Q_{r} \cap\left(\cup_{i=0}^{r-1} Q_{i}\right)$ is an $(n-1)$ manifold, and $K_{X}^{\prime \prime}=\left\{Q_{0}, \ldots, Q_{t}\right\}$ for some $t \geqq-1$.

If $C=\partial I^{n}, X=R_{l}$ or $X=\mathrm{cl}\left(R_{l} \backslash R_{l+1}\right)$ for some $l \geqq 0$, and it is easy to see that there is a sequence $\left\{Q_{0}, \ldots, Q_{s-1}\right\}$ satisfying (**). Therefore we now assume that $C$ is contained in some face $F$ of $I^{n}$.

The family $K_{k}(F)$, which contains $K_{X}^{\prime}$, forms a two-dimensional lattice, and we denote by $Q_{i j}^{1}$ the element in $i$-th row and $j$-th column. In like manner, $K_{X}^{\prime \prime}$ is contained in $\left\{\mathrm{cl}\left(Q \backslash\left(\cup K_{k}(F)\right)\right): Q \in K_{l}(F)\right\}$ which forms a two-dimensional lattice. Denote by $Q_{i j}^{0}$ the element in $i$-th row and $j$-th column. We can define a linear order in $K_{X}^{\prime} \cup K_{X}^{\prime \prime}$ by

(a) $Q_{i j}^{k}<Q_{i^{\prime} j^{\prime}}^{k^{\prime}}$ if $k<k^{\prime}$ or if $k=k^{\prime}$ and $i<i^{\prime}$,

(b) $Q_{i j}^{k}<Q_{i j^{\prime}}^{k}$ if $j<j^{\prime}$ unless $Q_{i-1, j}^{k} \leftarrow X$ and $Q_{i-1, j^{\prime}}^{k} \subset X$ in which case $Q_{i j^{\prime}}^{k}<Q_{i j}^{k}$.

Now we can enumerate $K_{X}^{\prime} \cup K_{X}^{\prime \prime}$ as $\left\{Q_{0}, \ldots, Q_{s-1}\right\}$ in such a way that $Q_{i}<Q_{j}$ if and only if $i<j$. It is a straightforward verification that (**) is true. Consequently (*) defines a partition of $K \mid X$ satisfying (v).

The proof of (vi) is similar. We use the above notation and again enumerate $K_{X}^{\prime} \cup K_{X}^{\prime \prime}$ as $\left\{Q_{0}, \ldots, Q_{s-1}\right\}$ in such a way that the right hand side of (*) defines a partition $K_{1}^{\prime}(X), \ldots, K_{s M}^{\prime}(X)$ of $K \mid X$ satisfying (vi). For this it is sufficient that (***) is true where $Q_{-1}=F_{0}^{\prime}(X)=\cup K_{0}^{\prime}(X)$ :

(***) For $r<s, \bigcup_{i=-1}^{r} Q_{i}$ is an n-manifold and $Q_{r} \cap\left(\cup_{i=-1}^{r-1} Q_{i}\right)$ is an $(n-1)$ manifold, and $K_{X}^{\prime}=\left\{Q_{n}, \ldots, Q_{t}\right\}$ for some $t \geqq-1$.

As above, we define a linear order in $K_{X}^{\prime} \cup K_{X}^{\prime \prime}$ by

(a') $Q_{i j}^{k}<Q_{i^{\prime} j^{\prime}}^{k^{\prime}}$ if $k>k^{\prime}$ or if $k=k^{\prime}$ and $i<i^{\prime}$,

(b') $Q_{i j}^{k}<Q_{i j^{\prime}}^{k}$ if $j<j^{\prime}$ unless $Q_{i+1, j}^{k} \subset X$ and $Q_{i+1, j^{\prime}}^{k} \leftarrow X$ in which case $Q_{i j^{\prime}}^{k}<Q_{i j}^{k}$.

Conclusion is as above.

8. Proof of Theorem 1. We can assume that either $C=\partial I^{n}$ or that $C$ is contained in some face $F$ of $I^{n}$. Otherwise, if $C \neq \partial I^{n}$, there is a PL homeomorphism $h: I^{n} \rightarrow I^{n}$ such that $h(C) \subset F$ and we replace $f$ by $f h^{-1}$.

In these circumstances we can use the above lemma. Let $M_{0} \supset M_{1} \supset \ldots$ be the sequence of the lemma. We use only the portion $M_{0} \supset M_{1} \supset M_{2} \supset M_{3}$ of the sequence and assume that $f \mid M_{1}$ is a lipeomorphism. Then we find a partition $K_{1}\left(M_{3}\right), \ldots, K_{m}\left(M_{3}\right)$ of $K \mid M_{3}$ as in (v) of the lemma. Define numbers $\delta_{1} \leqq \ldots \leqq \delta_{m}$ 
as in [5, 2.8] with $L=\operatorname{bilip}\left(f \mid M_{1}\right)$ and $M=m$. Now Lemma 2.9 of [5] remains true after the substitutions, when $\varepsilon^{\prime}>0$,

$$
L \mapsto \operatorname{bilip}\left(f \mid M_{1}\right), \quad M \mapsto m, \quad F_{r} \mapsto F_{r}\left(M_{3}\right), \quad K_{r}^{*} \mapsto K_{r}^{*}\left(M_{3}\right), \quad \varepsilon \mapsto \varepsilon^{\prime},
$$

even if the assumption that $f$ is a lipeomorphism $I^{n} \rightarrow f\left(I^{n}\right)$ of Lemma 2.9 is weakened into the assumption that bilip $\left(f \mid M_{1}\right)<\infty$.

Once this modification of Lemma 2.9 of [5] is proved, one can show as in 2.10 of [5], if $\varepsilon^{\prime}>0$ is given:

There is an embedding $f_{3}: M_{3} \rightarrow R^{n}$ such that

(i) $f_{3}\left|M_{3} \cap \partial I^{n}=f\right| M_{3} \cap \partial I^{n}$,

(ii) $\left\|f_{3}(x)-f(x)\right\| \leqq \varepsilon^{\prime} \lambda_{Q}$ if $Q \in K$ and $x \in Q \subset M_{3}$,

(iii) $f_{3} \mid M_{3} \cap$ int $I^{n}$ is PL,

(iv) $f_{3}$ is locally bilipschitz at points of $C$.

The next step is to consider the set $M_{0}^{\prime}=\operatorname{cl}\left(M_{0} \backslash M_{2}\right)=\mathrm{cl}\left(I^{n} \backslash M_{2}\right)$. Let $\varepsilon^{\prime \prime}: M_{0}^{\prime} \rightarrow[0, \infty)$ be a continuous function such that $\varepsilon^{\prime \prime}(x)>0$ if $x \in M_{0}^{\prime} \cap \operatorname{int} I^{n}$. Then we have:

There is an embedding $f_{0}^{\prime}: M_{0}^{\prime} \rightarrow R^{n}$ such that

(i') $f_{0}^{\prime}\left|M_{0}^{\prime} \cap \partial I^{n}=f\right| M_{0}^{\prime} \cap \partial I^{n}$,

(ii') $\left\|f_{0}^{\prime}(x)-f(x)\right\| \leqq \varepsilon^{\prime \prime}(x)$ if $x \in M_{0}^{\prime}$,

(iii') $f_{0}^{\prime} \mid M_{0}^{\prime} \cap$ int $I^{n}$ is PL.

This follows e.g. from Theorem 1 of Moise [2, Section 36]. (If this Theorem is used it is better first to seek an approximation for $f \mid\left(I^{n} \backslash M_{3}\right)$ and then restrict it to $\left.M_{0}^{\prime}\right)$.

We finish the proof by glueing $f_{3}$ and $f_{0}^{\prime}$ together by finding a function $f_{2}^{\prime}: M_{2}^{\prime}=$ $\operatorname{cl}\left(M_{2} \backslash M_{3}\right) \rightarrow R^{n}$ such that

(i") $f_{3}, f_{0}^{\prime}$ and $f_{2}^{\prime}$ define together an embedding $I^{n} \rightarrow R^{n}$,

(ii") $f_{2}^{\prime}\left|M_{2}^{\prime} \cap \partial I^{n}=f\right| M_{2}^{\prime} \cap \partial I^{n}$,

(iii") $\left\|f_{2}^{\prime}(x)-f(x)\right\| \leqq \varepsilon$ if $x \in M_{2}^{\prime}$,

(iv") $f_{2}^{\prime} \mid M_{2}^{\prime} \cap \operatorname{int} I^{n}$ is PL.

If we have found such a function $f_{2}^{\prime}$, then $f^{*}=f_{0}^{\prime} \cup f_{2}^{\prime} \cup f_{3}$ satisfies the conditions of Theorem 1, provided that $\varepsilon^{\prime}$ and $\varepsilon^{\prime \prime}$ are sufficiently small.

Since $f \mid M_{1}$ is a lipeomorphism we can again use the construction of $[5,2.11]$ to prove the existence of such $f_{2}^{\prime}$. Let $K_{0}^{\prime}\left(M_{2}^{\prime}\right)$ be as in (vi) of the above lemma and let $K_{1}^{\prime}\left(M_{2}^{\prime}\right), \ldots, K_{m^{\prime}}^{\prime}\left(M_{2}^{\prime}\right)$ be the partition of $K \mid M_{2}^{\prime}$ in (vi) of the lemma. Since bilip $\left(f \mid M_{1}\right)<\infty$ we can apply Lemma 2.6 of [5] and find numbers $\delta_{0} \leqq \ldots \leqq \delta_{m^{\prime}}$ as in 2.8, with $L=$ bilip $\left(f \mid M_{1}\right)$. Now, assuming that $\varepsilon^{\prime}$ and $\varepsilon^{\prime \prime}$ are sufficiently small, we can extend $f_{0}^{\prime} \cup f_{3}$ (which is defined in a set containing $F_{0}^{\prime}\left(M_{2}^{\prime}\right)$ ) first to $F_{1}^{\prime}\left(M_{2}^{\prime}\right)$, then to $F_{2}^{\prime}\left(M_{2}^{\prime}\right), \ldots$, and finally to $F_{m^{\prime}}^{\prime}\left(M_{2}^{\prime}\right)$ as in $[5,2.11]$. Notice that the situation 
is now simpler since we do not require (i) of Lemma 2.9, (ii) being sufficient. As in 2.10 , it is shown that we obtain in this manner an embedding $f_{2}^{\prime}: M_{2}^{\prime} \rightarrow R^{n}$ (which need not be a LIP embedding) satisfying (i")-(iv").

9. Proof of Theorem 2. We can assume $\varepsilon$ to be chosen in such a way that, for each $x \in M$, the set $\{y \in N: d(y, f(x)) \leqq \varepsilon(x)\}$ is contained in a set lipeomorphic to $R^{n}$ (or to $R_{+}^{n}$ if $x \in \partial M$ ). We can find a family $\mathscr{V}$ of closed LIP $n$-balls in $M$ such that $\mathscr{V}$ is locally finite in $M, \cup \mathscr{V} \subset M \backslash C$, and $\cup$ int $V: V \in \mathscr{V}\} \supset M \backslash U$. There are also continuous functions $\eta_{i}: M \rightarrow(0, \infty), 0 \leqq i \leqq k$, where $k$ depends only on $n$, such that:

(1) $\eta_{0}+\ldots+\eta_{k-1} \leqq \eta_{k}=\varepsilon$.

(2) If $x \in \partial V, V \in \mathscr{V}, x$ has a neighbourhood $U_{x}$ such that $\left(U_{x}, U_{x} \cap \partial V\right)$ is lipeomorphic to $\left(R^{n}, R^{n-1}\right)$ or to $\left(R_{+}^{n}, R_{+}^{n-1}\right)$, according to whether $x \notin \partial M$ or $x \in \partial M$.

(3) There is a partition $\mathscr{V}_{1}, \ldots, \mathscr{V}_{k}$ of $\mathscr{V}$ such that each $\mathscr{V}_{i}$ consists of disjoint balls.

(4) Let $V \in \mathscr{V}$ and $x \in V$. Then $\operatorname{diam} f(V) \leqq \eta_{0}(x)$.

(5) If $V \in \mathscr{V}$ and $i<k, 3\left(\max _{x \in V} \eta_{i}(x)\right) \leqq \min _{x \in V} \eta_{i+1}(x)$.

It is not difficult to see that there is such a family $\mathscr{V}$ and such functions $\eta_{i}$. We give only some hints for (3). Choose first an open cover $\mathscr{U}$ of $M \backslash U$ such that the elements of $\mathscr{U}$ have a sufficiently small diameter. In addition we assume that $\mathscr{U}$ is locally finite and that there is a partition $\mathscr{U}_{1}, \ldots, \mathscr{U}_{n+1}$ of $\mathscr{U}$ such that each $\mathscr{U}_{i}$ consists of disjoint sets and that $\cup \mathscr{U}_{i}$ is lipeomorphic to an open subset of $R_{+}^{n}$. We can also assume that there is a cover $\mathscr{U}^{\prime}=\left\{C_{V}: V \in \mathscr{U}\right\}$ of $M \backslash U$ whose elements are compact sets with $C_{V} \subset V$. Let $C_{i}=\cup\left\{C_{V}: V \in \mathscr{U}_{i}\right\}$. Since now a neighbourhood of $C_{i}$ is lipeomorphic to an open subset of $R_{+}^{n}$, we can find a locally finite (in $M$ ) cover $\mathscr{V}^{i}$ of $C_{i}$ such that the elements of $\mathscr{V}^{i}$ are closed LIP $n$-balls (with $\cup$ int $\left.\left.V: V \in \mathscr{V}^{i}\right\} \supset C_{i}\right)$ and such that there is a partition $\mathscr{V}_{(i-1)(n+1)+1}, \ldots, \mathscr{V}_{i(n+1)}$ of $\mathscr{V}^{i}$ where each $\mathscr{V}_{j}$ consists of disjoint $n$-balls. Then $\mathscr{V}=\mathscr{V}_{1} \cup \ldots \cup \mathscr{V}_{(n+1)^{2}}$ is the desired cover of $M \backslash U$. This construction gives $k=(n+1)^{2}$ but most certainly we could have $k=n+1$.

We can also find for each $V \in \mathscr{V}$ a closed LIP $n$-ball $W_{V} \subset$ int $V$ such that \{int $\left.W_{V}: V \in \mathscr{V}\right\}$ still covers $M \backslash U$. Let $V^{*} \subset U$ be some closed set such that (int $\left.V^{*}\right\} \cup\left\{\right.$ int $\left.W_{V}: V \in \mathscr{V}\right\}$ is a cover of $M$. (Then int $V^{*} \supset C$.) Let $\mathscr{V}_{0}=\left\{V^{*}\right\}$ and set $W_{V^{*}}=V^{*}$.

We prove Theorem 2 by showing:

There is a sequence $f=f_{0}, f_{1}, \ldots, f_{k}$ of homeomorphisms $M \rightarrow N$ such that:

(i) Each $f_{i}$ is locally bilipschitz in $\cup\left\{W_{V}: V \in \mathscr{V}_{j}\right.$ with $\left.j \leqq i\right\}$.

(ii) $\operatorname{diam} f_{i}(V) \leqq \eta_{i}(x)$ if $x \in V \in \mathscr{V}, \quad 0 \leqq i \leqq k$.

(iii) $d\left(f_{i}(x), f_{i-1}(x)\right) \leqq \eta_{i-1}(x)$ for $\quad x \in M \quad$ and $0<i \leqq k$.

(iv) If $x \in M \backslash\left(\cup \mathscr{V}_{i}\right), f_{i}(x)=f_{i-1}(x)$ for $0<i \leqq k$. 
We have already set $f_{0}=f$. Assume that we have defined $f_{j}$ for $0 \leqq j \leqq m-1$ satisfying (i)-(iv). Choose some ball $V \in \mathscr{V}_{m}$. By (ii) (since $\eta_{m-1} \leqq \varepsilon$ ) there is some set $W \subset N$ containing $f_{m-1}(V)$ and a lipeomorphism $\psi: W \rightarrow R^{n}$. There is also a lipeomorphism $\varphi: V \rightarrow I^{n}$. Then $\psi \circ f_{m-1} \circ \varphi^{-1}: I^{n} \rightarrow R^{n}$ is an embedding which is locally bilipschitz at $x \in I^{n}$ if $f_{m-1}$ is locally bilipschitz at $\varphi^{-1}(x) \in V$. Let $C^{\prime}=$ $\varphi\left(\partial V \cap\left(\cup\left\{W_{V}: V \in \mathscr{V}_{j}\right.\right.\right.$ with $\left.\left.\left.j<m\right\}\right)\right)$. Then $C^{\prime} \subset \partial I^{n}$ and, by (i), $\psi \circ f_{m-1} \circ \varphi^{-1}$ is locally bilipschitz in $C^{\prime}$. Therefore we may apply Theorem 1 and find an embedding $f^{*}: I^{n} \rightarrow R^{n}$ with the same boundary values as $\psi \circ f_{m-1} \circ \varphi^{-1}$ that is PL in int $I^{n}$ and locally bilipschitz in $C^{\prime}$. Therefore $f_{V}^{*}=\psi^{-1} \circ f^{*} \circ \varphi: V \rightarrow N$ is locally bilipschitz in $\cup\left\{W_{V^{\prime}} \cap V: V^{\prime} \in \mathscr{V}_{j}\right.$ with $\left.j<m\right\} \cup$ int $V$.

We choose such a mapping $f_{V}^{*}: V \rightarrow N$ for each $V \in \mathscr{V}_{m}$. Now we can define

$$
f_{m}(x)= \begin{cases}f_{V}^{*}(x) & \text { if } x \in V \in \mathscr{V}_{m}, \\ f_{m-1}(x) & \text { otherwise. }\end{cases}
$$

Since for each $V \in \mathscr{V}_{m}, f_{V}^{*}\left|\partial V=f_{m-1}\right| \partial V, f_{m}$ is a homeomorphism $M \rightarrow N$. Let $V \in \mathscr{V}_{m}$ and let $x \in \partial V \cap\left(\cup\left\{W_{V}: V \in \mathscr{V}_{j}\right.\right.$ with $\left.\left.j<m\right\}\right)$. Then $f_{m-1}$ is locally bilipschitz at $x$, as is $f_{m} \mid V$. Therefore, since $\mathscr{V}$ is locally finite, $f_{m}$ is locally Lipschitz at $x$. Since $f_{m-1}(\partial V)$ has a local LIP collar at $f_{m-1}(x)$, also $f_{m}^{-1}$ is locally Lipschitz at $f_{m-1}(x)=f_{m}(x)$. Thus $f_{m}$ is locally bilipschitz in $\cup\left\{W_{V}: V \in \mathscr{V}_{j}, j \leqq m\right\}$. We have shown that $f_{m}$ satisfies (i). Clearly, it satisfies (iii), (iv), and, by (5), also (ii).

Consider the function $g=f_{k}$. Since $(\cup \mathscr{V}) \cap C=\emptyset$ and $f_{0}=f$, we have by (iv) $g|C=f| C$. By (i), $g$ is a lipeomorphism $M \rightarrow N$. By (iii) and (1), $d(g(x)$, $f(x)) \leqq \varepsilon(x)$ for $x \in M$. Thus $g$ is the lipeomorphism $M \rightarrow N$ sought for.

10. Proof of Theorem 3. We first show that we may assume $f \mid I^{n} \backslash X$ to be a LIP embedding where $X=\left\{x \in \partial I^{n}: f\right.$ is not locally bilipschitz at $\left.x\right\}$. Then $X$ is a closed subset of $\partial I^{n}$.

Let $A=\partial I^{n} \backslash X$ and let $U$ be an open subset of $I^{n}$ containing $A$ such that $f \mid U$ is a lipeomorphism $U \rightarrow f(U)$ and let $C \subset U$ be a closed neighbourhood of $A$ in $I^{n} \backslash X$. We regard int $I^{n}$ and $f$ (int $I^{n}$ ) as LIP-manifolds, the LIP-structures being those of open submanifolds of $R^{n}$. Let $\varepsilon^{\prime}:$ int $I^{n} \rightarrow(0, \varepsilon / 2)$ be a continuous function such that if $h$ : int $I^{n} \rightarrow R^{n}$ is a continuous function and $\|h(x)-f(x)\|<\varepsilon^{\prime}(x)$ for $x \in$ int $I^{n}$, then we can extend $h$ to a continuous function $I^{n} \rightarrow R^{n}$ by setting $h\left|\partial I^{n}=f\right| \partial I^{n}$. Now we can apply Theorem 2 with the substitutions

$$
M \mapsto \operatorname{int} I^{n}, \quad N \mapsto f\left(\text { int } I^{n}\right), \quad U \mapsto U \cap \text { int } I^{n}, \quad C \mapsto C \cap \text { int } I^{n}, \quad \varepsilon \mapsto \varepsilon^{\prime} .
$$

Let $g^{\prime}$ : int $I^{n} \rightarrow f$ (int $I^{n}$ ) be the lipeomorphism obtained. Then we can extend $g^{\prime}$ to a continuous map $g: I^{n} \mapsto R^{n}$ by setting $g\left|\partial I^{n}=f\right| \partial I^{n}$. But then $g$ must be an embedding $I^{n} \rightarrow R^{n}$ such that $g \mid I^{n} \backslash X$ is a LIP embedding. Clearly, if $f^{*}$ is the map given by Theorem 3 with substitutions $\varepsilon \mapsto \varepsilon / 2$ and $f \mapsto g$, then $f^{*}$ satisfies the conditions of Theorem 3 also with respect to the original $f$ and $\varepsilon$.

We now assume that $f \mid I^{n} \backslash X$ is a lipeomorphism onto $f\left(I^{n} \backslash X\right)$. As in the proof of Theorem 1, we can assume that $X$ is contained in some face $F$ of $I^{n}$, other- 
wise either $X=\partial I^{n}$ and there is nothing to prove in addition to Theorem 1, or we can replace $f$ by another map. Let $M_{0} \supset M_{1} \supset \ldots$ be the sequence of the lemma of Section 7 where we replace $C$ by $X$. Then $\operatorname{bilip}\left(f \mid \mathrm{cl}\left(M_{i} \backslash M_{i+1}\right)\right)<\infty$ for $i \geqq 0$.

Let $M^{\prime}=\bigcup_{i \geqq 0} \operatorname{cl}\left(M_{2 i} \backslash M_{2 i+1}\right)$ and let $M^{\prime \prime}=\bigcup_{i \geqq 0} \operatorname{cl}\left(M_{2 i+1} \backslash M_{2 i+2}\right)$. Then $I^{n} \backslash X=M^{\prime} \cup M^{\prime \prime}$. Let $\varepsilon_{i}>0$ be given for $i=0,2,4, \ldots$. We can now apply separately to each subset $\operatorname{cl}\left(M_{2 i} \backslash M_{2 i+1}\right)$ of $M^{\prime}$ the method of [5] in 2.10 and 2.11 using (v) of the lemma of Section 7 to obtain a map $f^{\prime}: M^{\prime} \rightarrow R^{n}$ such that

(i) $f^{\prime} \mid \operatorname{cl}\left(M_{2 i} \backslash M_{2 i+1}\right) \quad$ is a LIP embedding, $i \geqq 0$,

(ii) $\left\|f^{\prime}(x)-f(x)\right\| \leqq \varepsilon_{2 i} \lambda_{Q} \quad$ if $\quad x \in Q \in K$ and $Q \subset \mathrm{cl}\left(M_{2 i} \backslash M_{2 i+1}\right), \quad i \geqq 0$,

(iii) $f^{\prime}\left|M^{\prime} \cap \partial I^{n}=f\right| M^{\prime} \cap \partial I^{n}$,

(iv) $f^{\prime} \mid M^{\prime} \cap \operatorname{int} I^{n}$ is PL,

(v) If $i \geqq 0, \quad\left\{\beta_{Q} f^{\prime} \alpha_{Q}\left|2 I^{n} \cap \alpha_{Q}^{-1} M^{\prime}: Q \in K\right| \mathrm{cl}\left(M_{2 i+1} \backslash M_{2 i+2}\right)\right\} \quad$ is finite.

If the $\varepsilon_{i}$ 's are suitably chosen, $f^{\prime}$ is an embedding. Again, given $\varepsilon_{i}>0, i=1,3, \ldots$, and assuming that $\varepsilon_{i}: \mathrm{s}, i=0,2, \ldots$, are sufficiently small, we can find as above, using (vi) of the lemma of Section 7, a map $f^{\prime \prime}: M^{\prime \prime} \rightarrow R^{n}$ that satisfies conditions similar to (i)-(iv) and such that $f^{\prime}$ and $f^{\prime \prime}$ together define a LIP embedding $f_{0}: M^{\prime} \cup M^{\prime \prime}=I^{n} \backslash X \rightarrow R^{n}$ (resembling the construction of $f_{2}^{\prime}$ in the proof of Theorem 1). If $\varepsilon_{i} \rightarrow 0$ sufficiently fast as $i \rightarrow \infty$, we can extend $f_{0}$ to an embedding $f^{*}: I^{n} \rightarrow R^{n}$ with the desired properties.

\section{References}

[1] LuUkKainen, J., and J. VÄıs̈̈LÄ: Elements of Lipschitz topology. - Ann. Acad. Sci. Fenn. Ser. A I 3, 1977, 85-122.

[2] MoIse, E. E.: Geometric topology in dimensions 2 and 3. - Springer-Verlag, New York-Heidelberg-Berlin, 1977.

[3] SoRvali, T.: The boundary mapping induced by an isomorphism of covering groups. - Ann. Acad. Sci. Fenn. Ser. A I 526, 1972, 1-31.

[4] Sullivan, D.: Hyperbolic geometry and homeomorphisms. - To appear in the Proceedings of the Georgia Topology Conference 1977.

[5] VÄıÏ̈LÄ, J.: Piecewise linear approximation of lipeomorphisms. - Ann. Acad. Sci. Fenn. Ser. A I 3, 1977, 377-383.

University of Helsinki

Department of Mathematics

SF-00100 Helsinki 10

Finland

Received 7 March 1978

Revision received 19 May 1978 